\title{
3rd ICTs and Society Meeting; Paper Session - Inequalities: social, economic and political; Paper 6: Implications and precisions about digital exclusion in the UK and Chile
}

\author{
${ }^{1}$ Ellen J. Helsper \& ${ }^{2}$ Sergio Godoy-Etcheverry \\ ${ }^{1}$ London School of Economics and Political Science \\ ${ }^{2}$ School of Communications, P. Universidad Catolica de Chile
}

Acknowledgement: A study supported by The Conference Board, NY

As the internet and other digital technologies become increasingly massified, concerns about exclusion and the digital divide needs to go beyond the physical access/not access dichotomy, because it does not take into account the complexities involved on how and why technology is appropriated and integrated by persons to their everyday life and, ultimately, to enhance human welfare. Even the definition of the so-called decisional divide, which separates those who want technology from those who reject it does not necessarily account for the reasons, attitudes, fears or pressures behind this decision, which can change along time as well. This study wants to contribute to clarify this problem by exploring in depth how a specific, yet significant segment of population from two countries which lead ICT diffusion in their respective region, the UK and Chile, feels more or less integrated to technology, and what the motivators and obstacles, real or imaginary, they face. Perceptions of usefulness and purpose within a complex network of social ties are therefore key to understand the phenomenon.

Theoretically we assume technological engagement is related to engagement to social networks. We start by analysing secondary quantitative and institutional sources to compare and situate these two countries within the international context of socio-economic and technological development, and then we discuss the qualitative findings drawn from group discussions conducted in London and
Santiago de Chile in the last months of 2009 and early 2010. Middle and lower-middle class parents of children attending school and/or college were selected since they lived in environments where ICTs were widely used and therefore the pressure to use them was likely to be high. Furthermore, they constituted that grey area of digital inclusion/exclusion because they were neither too rich nor too poor, nor too old nor to young. We found that in both countries weaker social networks did seem to lead to less extensive engagement with ICTs, among other similarities. At the same time, there were important country differences. Some of them can be related to different development levels while others seemed more cultural, yet it became clear that socio-economic and development indicators are neither the only nor the best explanations of what goes on in the long tail of digital exclusion where people are somewhat but definitely not totally engaging with ICTs.

\section{Key issues to be raised in the ses- sion:}

- Digital inclusion/exclusion is an aspect of social integration: technological engagement is related to engagement to social networks.

- Digital inclusion/exclusion needs to be studied in that extensive, grey area where 
groups who are neither too rich nor too poor, nor too old nor to young, lie.

- There are relevant differences between the countries analysed in terms of attitudes to ICTs, gender relations, family roles, and the relevance of work as a factor of usage of technology. Yet weaker social networks did seem to lead to less extensive engagement with ICTs, among other similarities.

- Considering the factors analysed and their implications, the two countries considered seem different to each other, rather than one more "advanced" than the other.

- Cell phones are widely pervasive and felt as vital as clothing, whereas internet is regarded as something different and less relevant by some groups. The distinction may blur in the future as access to the web through mobiles (or other portable gadgets) become more generalised.

- Apart from being a continuum between intense usage and infrequent usage (or non usage at all, which is increasingly rare), the digital divide is neither unique nor static: there may be many digital divides. The concept needs to be redefined as both technology and society change.

\section{Why they are important for ICTs and Society research}

- Digital inclusion/exclusion is a changing phenomenon, and new issues become relevant to understand it properly.

- As ICTs become more prevalent, and especially in developing countries which are counting that ICTs may help them leapfrog to higher stages of development, analysis about inclusion/exclusion should also consider groups or sectors that lie on a grey area of basic, "access-only" technology adoption which may be rather superficial.

- International comparisons about technology usage and appropriation can consider new ways of categorisation, as countries may be different rather than all ranked along a single criterion of "advancement" in merely economic or technological terms. 